\title{
STABLE SUBNORMS ON FINITE-DIMENSIONAL POWER-ASSOCIATIVE ALGEBRAS*
}

\author{
MOSHE GOLDBERG ${ }^{\dagger}$
}

\begin{abstract}
Let $\mathcal{A}$ be a finite-dimensional power-associative algebra over a field $\mathbb{F}$, either $\mathbb{R}$ or $\mathbb{C}$, and let $\mathcal{S}$, a subset of $\mathcal{A}$, be closed under scalar multiplication. A real-valued function $f$ on $\mathcal{S}$ is called a subnorm if $f(a)>0$ for all $0 \neq a \in \mathcal{S}$, and $f(\alpha a)=|\alpha| f(a)$ for all $a \in \mathcal{S}$ and $\alpha \in \mathbb{F}$. If in addition, $\mathcal{S}$ is closed under raising to powers, then a subnorm $f$ is said to be stable if there exists a positive constant $\sigma$ so that

$$
f\left(a^{k}\right) \leq \sigma f(a)^{k} \quad \text { for all } a \in \mathcal{S} \text { and } k=1,2,3, \ldots .
$$

The purpose of this paper is to provide an updated account of our study of stable subnorms on subsets of finite-dimensional power-associative algebras over $\mathbb{F}$. Our aim is to review and discuss some of the results in several previous papers, dealing with both continuous and discontinuous subnorms.
\end{abstract}

Key words. Finite-dimensional power-associative algebras, Norms, Subnorms, Submoduli, Stable subnorms, Minimal polynomial, Radius of an element in a finite-dimensional power-associative algebra.

AMS subject classifications. 15A60, 16B99, 17A05.

1. Examples of subnorms and submoduli. Let $\mathcal{A}$ be a finite-dimensional algebra over a field $\mathbb{F}$. Throughout this paper we shall assume that $\mathbb{F}$ is either $\mathbb{R}$ or $\mathbb{C}$. Further, we shall assume that $\mathcal{A}$ is power-associative, i.e., that the subalgebra generated by any one element of $\mathcal{A}$ is associative, hence ensuring that powers of each element in $\mathcal{A}$ are unambiguously defined.

Let $\mathcal{S}$, a subset of $\mathcal{A}$, be closed under scalar multiplication (i.e., $a \in \mathcal{S}$ and $\alpha \in \mathbb{F}$ imply $\alpha a \in \mathcal{S})$. Following [GL1], we call a real-valued function

$$
f: \mathcal{S} \rightarrow \mathbb{R}
$$

a subnorm on $\mathcal{S}$ if for all $a \in \mathcal{S}$ and $\alpha \in \mathbb{F}$,

$$
\begin{aligned}
& f(a)>0, \quad a \neq 0, \\
& f(\alpha a)=|\alpha| f(a) .
\end{aligned}
$$

${ }^{*}$ Received by the editors January 19, 2008. Accepted for publication July 21, 2008. Handling Editor: Robert Guralnick.

$\dagger$ Department of Mathematics, Technion - Israel Institute of Technology, Haifa 32000, Israel (goldberg@math.technion.ac.il). 
If in addition, $\mathcal{S}$ is closed under raising to powers (i.e., $a \in \mathcal{S}$ implies $a^{k} \in \mathcal{S}, k=$ $1,2,3, \ldots)$, then a subnorm $f$ on $\mathcal{S}$ is called a submodulus if

$$
f\left(a^{k}\right)=f(a)^{k} \quad \text { for all } a \in \mathcal{S} \text { and } k=1,2,3, \ldots .
$$

If $\mathcal{S}$ is closed under multiplication as well, we say that a submodulus $f$ on $\mathcal{S}$ is a modulus if $f$ is multiplicative, i.e.,

$$
f(a b)=f(a) f(b) \quad \text { for all } a, b \in \mathcal{S} .
$$

We recall that if $\mathcal{S}$, a subset of $\mathcal{A}$, is closed under scalar multiplication and under addition, then a real-valued function $N$ is a norm on $\mathcal{S}$ if for all $a, b \in \mathcal{S}$ and $\alpha \in \mathbb{F}$,

$$
\begin{aligned}
& N(a)>0, \quad a \neq 0, \\
& N(\alpha a)=|\alpha| N(a), \\
& N(a+b) \leq N(a)+N(b) .
\end{aligned}
$$

Thus, in our finite-dimensional context, a norm is a subadditive continuous subnorm on $\mathcal{S} .^{1}$

Examples of subnorms, submoduli and moduli are not hard to come by. For instance, viewing the complex numbers,

$$
\mathbb{C}=\{z=\alpha+i \beta: \alpha, \beta \in \mathbb{R}\},
$$

as a 2-dimensional algebra over the reals, we note that for each fixed $p, 0<p \leq \infty$,

$$
|z|_{p}=\left(|\alpha|^{p}+|\beta|^{p}\right)^{1 / p}, \quad z=\alpha+i \beta \in \mathbb{C},
$$

is a continuous subnorm on $\mathbb{C}$. Evidently, $|\cdot|_{p}$ is a norm if and only if $1 \leq p \leq \infty$, and a submodulus - in fact, a modulus - only for $p=2$ where we get

$$
|z| \equiv|z|_{2}=\sqrt{\alpha^{2}+\beta^{2}} .
$$

Similarly, considering the quaternions

$$
\mathbb{H}=\{q=\alpha+i \beta+j \gamma+k \delta: \alpha, \beta, \gamma, \delta \in \mathbb{R}\}, \quad i^{2}=j^{2}=k^{2}=i j k=-1,
$$

as a 4-dimensional algebra over $\mathbb{R}$, we observe that

$$
|q|_{p}=\left(|\alpha|^{p}+|\beta|^{p}+|\gamma|^{p}+|\delta|^{p}\right)^{1 / p}, \quad q=\alpha+i \beta+j \gamma+k \delta \in \mathbb{H},
$$

\footnotetext{
${ }^{1}$ Of course, a subnorm $f$ on a finite-dimensional algebra $\mathcal{A}$ is said to be continuous if it is continuous with respect to the (unique) finite-dimensional norm-topology on $\mathcal{A}$.
} 
is a continuous subnorm for $0<p \leq \infty$, a norm precisely for $1 \leq p \leq \infty$, and a modulus,

$$
|q| \equiv|q|_{2}=\sqrt{\alpha^{2}+\beta^{2}+\gamma^{2}+\delta^{2}}
$$

for $p=2$.

Addressing the real 8-dimensional alternative ${ }^{2}$ (but not associative) algebra of the octonions,

$$
\mathbb{O}=\left\{c=\gamma_{1}+\gamma_{2} e_{2}+\cdots+\gamma_{8} e_{8}: \gamma_{j} \in \mathbb{R}\right\},
$$

with its intricate multiplication rule (e.g., [Ba, CS]), we remark that, by analogy with the two previous cases,

$$
|c|_{p}=\left(\left|\gamma_{1}\right|^{p}+\cdots+\left|\gamma_{8}\right|^{p}\right)^{1 / p}, \quad c=\gamma_{1}+\gamma_{2} e_{2}+\cdots+\gamma_{8} e_{8} \in \mathbb{O},
$$

is a continuous subnorm for $0<p \leq \infty$, a norm if and only if $1 \leq p \leq \infty$, and a modulus,

$$
|c| \equiv|c|_{2}=\sqrt{\left|\gamma_{1}\right|^{2}+\cdots+\left|\gamma_{8}\right|^{2}}
$$

for $p=2$ (a fact that stems from the Eight Square Theorem, [D], which implies that $|c d|=|c||d|$ for all $c, d \in \mathbb{O})$.

In our next example we examine the spectral radius,

$$
\rho(A)=\max \{|\lambda|: \lambda \in \mathbb{C} \text { an eigenvalue of } A\},
$$

where $A$ belongs to $\mathbb{F}^{n \times n}$, the algebra of $n \times n$ matrices over $\mathbb{F}$ with the usual operations. Since $\rho$ vanishes on nonzero nilpotent matrices, it is not a subnorm on $\mathbb{F}^{n \times n}$. It is, however, a subnorm, in fact a continuous submodulus (but usually not a modulus), on any subset of $\mathbb{F}^{n \times n}$ which is void of nonzero nilpotent matrices and closed under scalar multiplication and under raising to powers - for instance, on $\mathcal{N}_{n}(\mathbb{F})$, the set of normal $n \times n$ matrices over $\mathbb{F}$.

Contrary to norms, subnorms and submoduli are often discontinuous. An example of such submoduli is given in [GGL], where the underlying set is again $\mathcal{N}_{n}(\mathbb{F})$. Indeed, putting

$$
\tau(A)=\min \{|\lambda|: \lambda \in \mathbb{C} \text { an eigenvalue of } A\}, \quad A \in \mathcal{N}_{n}(\mathbb{F}),
$$

we observe that

$$
g_{\kappa}(A)= \begin{cases}\rho(A)^{\kappa+1} \tau(A)^{-\kappa}, & \tau(A)>0, \\ \rho(A), & \tau(A)=0,\end{cases}
$$

\footnotetext{
${ }^{2}$ An algebra $\mathcal{A}$ is called alternative if the subalgebra generated by any two elements in $\mathcal{A}$ is associative; hence, an alternative algebra is power-associative.
} 
is a submodulus on $\mathcal{N}_{n}(\mathbb{F})$ for every real constant $\kappa$. When $\kappa=0$, we obtain the (continuous) spectral radius. When $\kappa \neq 0, g_{\kappa}$ is discontinuous, since for the normal matrix

$$
A_{\varepsilon}=\operatorname{diag}(1, \ldots, 1, \varepsilon), \quad \varepsilon>0
$$

we get

$$
\lim _{\varepsilon \rightarrow 0} g_{\kappa}\left(A_{\varepsilon}\right)= \begin{cases}\infty, & \kappa>0 \\ 0, & \kappa<0\end{cases}
$$

whereas $g_{\kappa}(\operatorname{diag}(1, \ldots, 1,0))=1$.

Discontinuous subnorms can be easily constructed on arbitrary finite-dimensional algebras (power-associative or not) with dimension at least 2. Indeed, [G3], let $\mathcal{A}$ be such an algebra, and let $f$ be a continuous subnorm on $\mathcal{A}$. Select an element $a_{0} \in \mathcal{A}$, $a_{0} \neq 0$, and let

$$
\mathbf{V}=\left\{\alpha a_{0}: \alpha \in \mathbb{F}\right\}
$$

be the linear subspace of $\mathcal{A}$ generated by $a_{0}$. Fix a real $\kappa, \kappa>1$, and define

$$
h_{\kappa}(a)= \begin{cases}\kappa f(a), & a \in \mathbf{V}, \\ f(a), & a \in \mathcal{A} \backslash \mathbf{V} .\end{cases}
$$

Then $h_{\kappa}$ is a subnorm on $\mathcal{A}$, which is discontinuous at $a_{0}$ since

$$
\lim _{\substack{a \rightarrow a_{0} \\ a \notin \mathbf{V}}} h_{\kappa}(a)=\lim _{\substack{a \rightarrow a_{0} \\ a \notin \mathbf{V}}} f(a)=f\left(a_{0}\right) \neq h_{\kappa}\left(a_{0}\right) .
$$

When $\operatorname{dim} \mathcal{A}=1, \mathcal{A}$ is of the form of $\mathbf{V}$ in (1.8). Hence, in this case it is clear that every subnorm on $\mathcal{A}$ is in fact a (continuous) norm.

To exhibit a subnorm which is discontinuous everywhere, consider the familiar functional equation

$$
\varphi(x+y)=\varphi(x)+\varphi(y), \quad x, y \in \mathbb{R}
$$

whose (real) solutions have been discussed in the literature for over a century (e.g., [Ham], [HLP, Section 3.20], [HR], [Bo, Section 20], and [GL2, Section 2]). It is well known that any solution of (1.10) satisfies

$$
\varphi(r x)=r \varphi(x) \text { for all rational } r \text { and real } x .
$$

Hence, the only continuous solutions of (1.10) are of the form

$$
\varphi(x)=x \varphi(1), \quad x \in \mathbb{R},
$$


where $\varphi(1)$ is an arbitrary real value. It is also known that equation (1.10) has discontinuous solutions, and that all such solutions are discontinuous everywhere and unbounded (both from below and above) on any interval in $\mathbb{R}$. Further, given a positive number $c$, one may select a discontinuous solution $\varphi$ with $\varphi(c)=0$; thus,

$$
\varphi(x+c)=\varphi(x)+\varphi(c)=\varphi(x), \quad x \in \mathbb{R},
$$

and so $\varphi$ can be chosen to be $c$-periodic. By (1.12), $c>0$ is a period of $\varphi$ if and only if $\varphi(c)=0$; hence, if $c$ is a period then, by (1.11), so is every rational multiple of $c$.

Aided by these facts, it was proven in Theorem 2.1(c) of [GL2] that if $f$ is a continuous subnorm (submodulus, modulus) on $\mathbb{C}$, the 2-dimensional real algebra of the complex numbers over the reals, and if $\varphi$ is a discontinuous $\pi$-periodic solution of (1.10), then

$$
g_{\varphi}(z)=f(z) e^{\varphi(\arg z)}, \quad z \in \mathbb{C},
$$

( $\arg z$ denoting the principal argument of $z$, i.e., $0 \leq \arg z<2 \pi$ and $\arg 0=0$ ) is a subnorm (submodulus, modulus) which is ubiquitously discontinuous on $\mathbb{C}$.

Similar pathological constructions, where the resulting subnorms and submoduli lack any shred of continuity, were obtained in [GL2] for the quaternions as well as for $\mathcal{N}_{n}(\mathbb{F})$.

2. Stable subnorms. We begin this section by recalling two elementary observations that pertain to continuous subnorms on closed sets.

Proposition 2.1. [GL1, Lemma 1.1]. Let $\mathcal{S}$, a closed subset of a finitedimensional power-associative algebra $\mathcal{A}$ over $\mathbb{F}$, be closed under scalar multiplication. Let $f$ and $g$ be continuous subnorms on $\mathcal{S}$. Then $f$ and $g$ are equivalent; i.e., there exist constants $\mu>0, \nu>0$, such that

$$
\mu g(a) \leq f(a) \leq \nu g(a) \quad \text { for all } a \in \mathcal{S} .
$$

Proposition 2.2. [GL1, Lemma 1.2]. Let $\mathcal{S}$, a closed subset of a finitedimensional power-associative algebra $\mathcal{A}$ over $\mathbb{F}$, be closed under scalar multiplication and under raising to powers. Let $f$ be a continuous subnorm on $\mathcal{S}$ and let $g$ be a continuous submodulus on $\mathcal{S}$. Then,

$$
\lim _{k \rightarrow \infty} f\left(a^{k}\right)^{1 / k}=g(a) \quad \text { for all } a \in \mathcal{S} .
$$

Note that $g$ in Proposition 2.2 need not be a norm, even when $f$ is. Further, as the limit in (2.2) is unique, we may register: 
Corollary 2.3. [GL1, Corollary 1.1]. Let $\mathcal{S}$, a closed subset of a finitedimensional power-associative algebra $\mathcal{A}$ over $\mathbb{F}$, be closed under scalar multiplication and under raising to powers. Let $g$ be a continuous submodulus on $\mathcal{S}$. Then $g$ is the only continuous submodulus on $\mathcal{S}$.

The definition of submodulus gives rise to another simple, yet basic result:

Proposition 2.4. [GGL, Proposition 3]. Let $\mathcal{S}$, a subset of a power-associative algebra $\mathcal{A}$ over $\mathbb{F}$, be closed under scalar multiplication and under raising to powers. If $\mathcal{S}$ contains nonzero nilpotent elements, then $\mathcal{S}$ has no submodulus.

This observation (which holds for finite- as well as for infinite-dimensional algebras) implies, for example, that $\mathbb{F}^{n \times n}$ has no submodulus.

After the preliminaries in this and the previous sections, we are finally ready to address the main theme of this paper. Let $\mathcal{S}$, a subset of $\mathcal{A}$, be closed under scalar multiplication and under raising to powers. Following [GL1], we say that a subnorm $f$ is stable on $\mathcal{S}$ if for some positive constant $\sigma$,

$$
f\left(a^{k}\right) \leq \sigma f(a)^{k} \quad \text { for all } a \in \mathcal{S} \text { and } k=1,2,3, \ldots .
$$

If (2.3) holds for $\sigma=1$, we say that $f$ is strongly stable. Hence, for instance, all submoduli on $\mathcal{S}$ are strongly stable.

It is not difficult to show that every finite-dimensional power-associative algebra has stable subnorms, even stable norms. This will readily follow by proving that every finite-dimensional algebra (power-associative or not) can be endowed with submultiplicative norms.

Indeed (compare [AGL, Theorem 1.3]), let $N$ be a norm on an arbitrary finitedimensional algebra $\mathcal{A}$, and let $\mu>0$ be a constant. Then obviously, $N_{\mu} \equiv \mu N$ is a norm too. Put

$$
\mu_{N}=\sup \{N(a b): N(a)=N(b)=1\}
$$

Since $N$ is continuous and $\mathcal{A}$ is finite-dimensional, an elementary compactness argument shows that $\mu_{N}<\infty$. Moreover, $\mu_{N}$ can be written as

$$
\mu_{N}=\max _{a, b \neq 0} \frac{N(a b)}{N(a) N(b)} .
$$

Hence, if $\mu \geq \mu_{N}$, then for all $a, b \in \mathcal{A}$,

$$
N_{\mu}(a b)=\mu N(a b) \leq \mu \mu_{N} N(a) N(b) \leq \mu^{2} N(a) N(b)=N_{\mu}(a) N_{\mu}(b) ;
$$

so $N_{\mu}$ is sub-multiplicative on $\mathcal{A}$, and we are done. 
We can now quote the main result in [GL1] which characterizes stable subnorms on closed subsets that contain no nonzero nilpotents:

Theorem 2.5. [GL1, Theorem 1.1(a)]. Let $\mathcal{S}$, a closed subset of a finitedimensional power-associative algebra $\mathcal{A}$ over $\mathbb{F}$, be closed under scalar multiplication and under raising to powers. Let $f$ be a continuous subnorm on $\mathcal{S}$, and let $g$ be a continuous submodulus on $\mathcal{S}$. Then $f$ is stable on $\mathcal{S}$ if and only if $f \geq g$ on $\mathcal{S}$.

Since a submodulus is always stable, Theorem 2.5 provides a second short proof of Corollary 2.3: If $g$ and $g^{\prime}$ are continuous submodulus on $\mathcal{S}$ then, by the theorem, we have $g \geq g^{\prime}$ and $g^{\prime} \geq g$, which forces the desired result.

Theorem 2.5 has another immediate consequence:

Corollary 2.6. [GL1, Corollary 1.2]. Let $\mathcal{A}, \mathcal{S}$ and $g$ be as in Theorem 2.5. Then $g$ is the smallest of all stable continuous subnorms on $\mathcal{S}$.

Using Corollary 2.3 we find, for example, that the modulus functions in (1.2), (1.4) and (1.6) are the only continuous submoduli on $\mathbb{C}, \mathbb{H}$ and $\mathbb{O}$, respectively. Hence, by Theorem 2.5, a continuous subnorm $f$ is stable on $\mathbb{C}, \mathbb{H}$ or $\mathbb{O}$ if and only if $f$ majorizes the corresponding modulus function; so in particular, the subnorms in (1.1), (1.3) and (1.5) are stable precisely when $0<p \leq 2$.

To further illustrate Theorem 2.5, let $\mu$ and $\nu$ be positive constants, and consider the weighted sup norm on $\mathbb{C}$,

$$
|z|_{\mu, \nu, \infty}=\max \{\mu|\alpha|, \nu|\beta|\}, \quad z=\alpha+i \beta \in \mathbb{C} .
$$

Since the only continuous submodulus on $\mathbb{C}$ is given by (1.2), Theorem 2.5 implies that this norm is stable if and only if

$$
\max \{\mu|\alpha|, \nu|\beta|\} \geq \sqrt{\alpha^{2}+\beta^{2}} \text { for all } \alpha, \beta \in \mathbb{R},
$$

which, by Theorem 3.1 in [GL1], is equivalent to the simple inequality

$$
\mu^{2} \nu^{2} \geq \mu^{2}+\nu^{2}
$$

A similar illustration is obtained by considering the weighted $l_{1}$ norm

$$
|z|_{\mu, \nu, 1}=\mu|\alpha|+\nu|\beta|, \quad z=\alpha+i \beta \in \mathbb{C},
$$

where as before, $\mu>0, \nu>0$, are fixed. Appealing again to Theorem 2.5, we find that this norm is stable on $\mathbb{C}$ if and only if

$$
\mu|\alpha|+\nu|\beta| \geq \sqrt{\alpha^{2}+\beta^{2}} \text { for all } \alpha, \beta \in \mathbb{R},
$$


a condition which, by Theorem 3.3 in [GL1], is equivalent to

$$
\mu \geq 1, \quad \nu \geq 1 .
$$

By Proposition 2.4, Theorem 2.5 does not apply to the case when $\mathcal{S}$ contain nonzero nilpotent elements. If $\mathcal{S}$ is closed and consists only of nilpotents, then the question of stability for continuous subnorms becomes a triviality:

Proposition 2.7. [GGL, Proposition 4]. Let $\mathcal{S}$, a closed subset of nilpotent elements in a finite-dimensional power-associative algebra $\mathcal{A}$ over $\mathbb{F}$, be closed under scalar multiplication and under raising to powers. Then all continuous subnorms on $\mathcal{S}$ are stable.

3. Stable subnorms on matrices. Since every finite-dimensional associative algebra over $\mathbb{F}$ is algebraically isomorphic to a matrix algebra over $\mathbb{F}$, we take special interest in $\mathbb{F}^{n \times n}$, the algebra of $n \times n$ matrices over $\mathbb{F}$ with the usual operations.

We recall that the spectral radius is a continuous submodulus on any subset of $\mathbb{F}^{n \times n}$ which is void of nonzero nilpotents and closed under scalar multiplication and under raising to powers. Combining this fact with Proposition 2.4 and Corollary 2.3, we obtain:

TheOrem 3.1. [GL1, Theorem 1.2]. Let $\mathcal{S}$, a subset of $\mathbb{F}^{n \times n}$, be closed under scalar multiplication and under raising to powers. Then:

(a) The set $\mathcal{S}$ has a submodulus if and only if $\mathcal{S}$ is void of nonzero nilpotents.

(b) If $\mathcal{S}$ is void of nonzero nilpotents, then $\rho$, the spectral radius, is a submodulus on $\mathcal{S}$.

(c) If $\mathcal{S}$ is closed and void of nonzero nilpotents, then $\rho$ is the only continuous submodulus on $\mathcal{S}$.

Just as for norms, we use standard nomenclature and say that a subnorm $f$ on a subset $\mathcal{S}$ of $\mathbb{F}^{n \times n}$ is spectrally dominant if $f$ majorizes the spectral radius, i.e.,

$$
f(A) \geq \rho(A) \text { for all } A \in \mathcal{S} .
$$

Hence, Theorems 2.5 and 3.1(c) yield:

TheOREm 3.2. [GL1, Theorem 1.3(a)]. Let $\mathcal{S}$, a closed subset of $\mathbb{F}^{n \times n}$, be void of nonzero nilpotents and closed under scalar multiplication and under raising to powers. Let $f$ be a continuous subnorm on $\mathcal{S}$. Then $f$ is stable on $\mathcal{S}$ if and only if $f$ is spectrally dominant on $\mathcal{S}$.

An illustration of the last two theorems is obtained by recalling that $\mathcal{N}_{n}(\mathbb{F})$, the closed set of normal $n \times n$ matrices over $\mathbb{F}$, is void of nonzero nilpotents and 
closed under scalar multiplication and under raising to powers. Thus, $\rho$ is the only continuous submodulus on $\mathcal{N}_{n}(\mathbb{F})$, and a continuous subnorm $f$ is stable on this set if and only if $f \geq \rho$ there.

The assumption in Theorems 3.1(c) and 3.2 that $\mathcal{S}$ is closed, cannot be dropped. This was established in [G1] by noting that $G L_{n}(\mathbb{F}) \cup\{0\}$, the union of the general linear group of $n \times n$ invertible matrices over $\mathbb{F}$ and the zero matrix, is not a closed subset of $\mathbb{F}^{n \times n}$, and that both $\rho$ and

$$
\tau(A)=\min \{|\lambda|: \lambda \in \mathbb{C} \text { an eigenvalue of } A\}
$$

are continuous submoduli on this set. Hence, $\rho$ is not the only continuous submodulus on $G L_{n}(\mathbb{F}) \cup\{0\}$, and $\tau$ is stable there without majorizing $\rho$.

We note that while by Theorem 3.1(c), $\rho$ is the only continuous submodulus on the closed set $\mathcal{S}$ in Theorem 3.2, such a set may have infinitely many discontinuous submoduli, as demonstrated by the action of $g_{\kappa}$ in $(1.7)$ on $\mathcal{N}_{n}(\mathbb{F})$.

The heart of Theorem 3.2 is the assertion that if a closed subset $\mathcal{S}$ of $\mathbb{F}^{n \times n}$ is void of nonzero nilpotents and closed under scalar multiplication and under raising to powers, and if $f$ is a continuous subnorm on $\mathcal{S}$, then spectral dominance implies stability. In the opposite direction one may ignore the issue of nilpotents and prove a little more:

Proposition 3.3. [GL2, Theorem 1.5(b)]. Let $\mathcal{S}$, a closed subset of $\mathbb{F}^{n \times n}$, be closed under scalar multiplication and under raising to powers. Let $f$ be a continuous stable subnorm on $\mathcal{S}$. Then $f$ is spectrally dominant on $\mathcal{S}$.

The converse of Proposition 3.3 is false; i.e., continuous spectrally dominant subnorms on subsets of $\mathbb{F}^{n \times n}$ which are closed under scalar multiplication and under raising to powers, may fail to be stable. In light of Theorem 3.2 and Proposition 2.7, such subsets of $\mathbb{F}^{n \times n}$ must contain, of course, both nonzero nilpotent matrices and matrices which are not nilpotent.

For example, [GGL], consider the 2-dimensional matrix algebra

$$
\mathcal{A}=\{\alpha I+\beta B: \alpha, \beta \in \mathbb{F}\}
$$

where $B$ is a fixed nonzero nilpotent matrix in $\mathbb{F}^{n \times n}$ with $B^{2}=0$. Define

$$
N(\alpha I+\beta B)=\max \{|\alpha|,|\beta|\}, \quad \alpha I+\beta B \in \mathcal{A} .
$$

Surely, $\mathcal{A}$ is a proper subalgebra of $\mathbb{F}^{n \times n}$ swarming with nonzero nilpotent matrices as well as with matrices which are not nilpotent. Further, $N$ is a spectrally dominant norm on $\mathcal{A}$. Yet, $(I+B)^{k}=1+k B$, so

$$
\lim _{k \rightarrow \infty} N\left((I+B)^{k}\right)=\infty
$$


which explodes the possibility that $N$ is stable on $\mathcal{A} .^{3}$

In view of the above example, it seems desirable to characterize the class of all subalgebras $\mathcal{A}$ of $\mathbb{F}^{n \times n}$ which contain nonzero nilpotent matrices as well as matrices which are not nilpotent, and which possess the property that a norm on $\mathcal{A}$ is stable if and only if it is spectrally dominant.

The celebrated Friedland-Zenger Theorem tells us that $\mathbb{C}^{n \times n}$ belongs to this class:

Theorem 3.4. [FZ, Theorem 1]. A norm on $\mathbb{C}^{n \times n}$ is stable if and only if it is spectrally dominant.

To illustrate Theorem 3.4, select an inner product on $\mathbb{C}^{n}$ and consider the corresponding numerical radius

$$
w(A)=\max \left\{|(A x, x)|: x \in \mathbb{C}^{n},(x, x)=1\right\}, \quad A \in \mathbb{C}^{n \times n} .
$$

It is not hard to prove (e.g., [Hal, Section 173], [GT]) that $w$ is a spectrally dominant norm on $\mathbb{C}^{n \times n}$. Hence, by Theorem 3.4, $w$ is stable. As a matter of fact, $w$ is strongly stable on $\mathbb{C}^{n \times n}$ —a remarkable result due to Berger, [Be], [P], [Hal, Section 176].

We conjecture that, just like in the complex case, a norm on $\mathbb{R}^{n \times n}$ is stable if and only if it is spectrally dominant. Unable to prove this conjecture, we can post simpler results relating to certain classes of norms on $\mathbb{R}^{n \times n}$. For example, we recall that a norm $N$ on $\mathbb{F}^{n \times n}$ is quasimonotone if it is monotone on the cone of matrices with positive entries, i.e.,

$$
0 \leq A \leq B \Rightarrow N(A) \leq N(B), \quad A, B \in \mathbb{F}^{n \times n},
$$

where the inequalities $0 \leq A \leq B$ are construed entrywise. With this definition, we can prove:

Theorem 3.5. [GL3, Theorem 3.3]. A quasimonotone norm on $\mathbb{R}^{n \times n}$ is stable if and only if it is spectrally dominant.

We also recall that a norm $N$ on $\mathbb{F}^{n \times n}$ is monotone if

$$
A^{+} \leq B^{+} \Rightarrow N(A) \leq N(B), \quad A, B \in \mathbb{F}^{n \times n},
$$

where $A^{+}$is the matrix obtained by taking the absolute values of the entries of $A$. So it follows that monotonicity implies quasimonotonicity. Further, $N$ is said to be absolute if

$$
N(A)=N\left(A^{+}\right) \text {for all } A \in \mathbb{F}^{n \times n} .
$$

\footnotetext{
${ }^{3}$ A similar example, exhibiting a closed subset $\mathcal{S}$ of $\mathbb{F}^{n \times n}$ which is not an algebra, and a continuous unstable spectrally dominant subnorm on $\mathcal{S}$ which is not a norm, can be found in [GGL, pp. 216-217].
} 
And it is well known (e.g., [Z, Theorem 108.1], [HJ, Theorem 5.5.10]; compare [BSW]) that a norm on $\mathbb{F}^{n \times n}$ is monotone if and only if it is absolute. Thus, Theorem 3.5 implies:

Corollary 3.6. [GGL, Theorem 5]. An absolute norm on $\mathbb{R}^{n \times n}$ is stable if and only if it is spectrally dominant.

To conclude this section we point out that the Friedland-Zenger theorem cannot be extended to subnorms, not even to continuous subnorms. More precisely, we assert that not all continuous spectrally dominant subnorms on $\mathbb{C}^{n \times n}$ are stable.

Indeed (compare [GL3, Section 3]), let $N$ be a norm on $\mathbb{C}^{n \times n}$, and define

$$
f(A)=\max \left\{\rho(A), N\left(A-D_{A}\right)\right\}, \quad A \in \mathbb{C}^{n \times n},
$$

where $D_{A}$ is the diagonal part of $A$. Clearly, $f$ is a continuous spectrally dominant subnorm on $\mathbb{C}^{n \times n}$. Let $J_{\kappa}$ be the $n \times n$ matrix all of whose entries are zero, except for its upper-right entry, $\kappa$, which is chosen so that $N\left(J_{\kappa}\right)=1$. Then for $A_{\kappa}=I+J_{\kappa}$ we have $f\left(A_{\kappa}\right)=1$. Yet,

$$
\lim _{k \rightarrow \infty} f\left(A_{\kappa}^{k}\right)=\lim _{k \rightarrow \infty} f\left(I+k J_{\kappa}\right)=\lim _{k \rightarrow \infty} N\left(k J_{\kappa}\right)=\infty,
$$

so $f$ is not stable on $\mathbb{C}^{n \times n}$. Of course, $f$ is not a norm on $\mathbb{C}^{n \times n}$; for if it were, then by the Friedland-Zenger Theorem, it would be stable.

4. Subnorms and radii. Let $a$ be an element of a finite-dimensional powerassociative algebra over $\mathbb{F}$. As usual, by a minimal polynomial of $a$, we mean a monic polynomial of lowest positive degree with coefficients in $\mathbb{F}$ that annihilates $a$. With this familiar definition, and taking into account that $\mathcal{A}$ may or may not have a unit, one can prove:

Theorem 4.1. [G2, Theorem 1.1(a)]. Let $\mathcal{A}$ be a finite-dimensional powerassociative algebra over $\mathbb{F}$. Then every element a in $\mathcal{A}$ possesses a unique minimal polynomial.

As pointed out in $[\mathrm{G} 2], p_{a}$, the minimal polynomial of $a$, may depend not only on $a$, but also on the underlying algebra. For example, fix an idempotent matrix $M \in \mathbb{F}^{n \times n}, M \neq I$, and consider the matrix algebra

$$
\mathcal{A}=\left\{M A M: A \in \mathbb{F}^{n \times n}\right\}
$$

with the usual operations. Surely, $M$ belongs to $\mathcal{A}$. Further, $M$ is the unit in $\mathcal{A}$; hence the minimal polynomial of $M$ in $\mathcal{A}$ is $p_{M}(t)=t-1$. On the other hand, since the unit in $\mathbb{F}^{n \times n}$ is $I$, it is not hard to verify that the minimal polynomial of $M$ in $\mathbb{F}^{n \times n}$ is $q_{M}(t)=t^{2}-t$. 
This example, as well as others which can be found in [G2], are but special cases of a more general phenomenon:

Theorem 4.2. [G2, Theorem 1.2]. Let $\mathcal{A}$ and $\mathcal{B}$ be finite-dimensional powerassociative algebras over $\mathbb{F}$, such that $\mathcal{A}$ is a subalgebra of $\mathcal{B}$. Let a be an element of $\mathcal{A}$, and let $p_{a}$ and $q_{a}$ denote the minimal polynomials of a in $\mathcal{A}$ and in $\mathcal{B}$, respectively. Then either $p_{a}=q_{a}$ or $q_{a}(t)=t p_{a}(t)$.

Having established the existence and uniqueness of $p_{a}$, the minimal polynomial of an element $a$ in $\mathcal{A}$, we proceed to define the radius of $a$ as

$$
r(a)=\max \left\{|\lambda|: \lambda \in \mathbb{C} \text { a root of } p_{a}\right\} .
$$

We emphasize that, unlike the minimal polynomial of $a$, the radius $r(a)$ is independent of $\mathcal{A}$ in the sense that if $\mathcal{B}$ is another finite-dimensional power-associative algebra over $\mathbb{F}$, such that $\mathcal{A}$ is contained in $\mathcal{B}$, then the radii of a in $\mathcal{A}$ and in $\mathcal{B}$ coincide.

The proof of this assertion, given in [G2], is short: By Theorem 4.2, $p_{a}$ and $q_{a}$, the minimal polynomials of $a$ in $\mathcal{A}$ and in $\mathcal{B}$, are either identical or satisfy $q_{a}(t)=t p_{a}(t)$. Hence, the nonzero roots of $p_{a}$ and $q_{a}$ are equal; so

$$
\max \left\{|\lambda|: \lambda \in \mathbb{C} \text { a root of } p_{a}\right\}=\max \left\{|\lambda|: \lambda \in \mathbb{C} \text { a root of } q_{a}\right\}
$$

and the proof is complete.

In view of the above assertion, and since the roots of the minimal polynomial of a matrix $A \in \mathbb{F}^{n \times n}$ are its eigenvalues, we get:

Proposition 4.3. [G2, (2.2)]. If $A$ belongs to a subalgebra of $\mathbb{F}^{n \times n}$, then

$$
r(A)=\rho(A)
$$

where $\rho$ denotes the spectral radius.

As it is, the radius $r$ retains some of the familiar properties of the spectral radius not only for matrices, but in general as well:

Theorem 4.4. [G2, Theorems 2.1 and 2.4]. Let $\mathcal{A}$ be a finite-dimensional powerassociative algebra over $\mathbb{F}$. Then:

(a) The radius $r$ is a nonnegative function on $\mathcal{A}$.

(b) For all $a \in \mathcal{A}$ and $\alpha \in \mathbb{F}$,

$$
r(\alpha a)=|\alpha| r(a)
$$


(c) For all $a \in \mathcal{A}$ and all positive integers $k$,

$$
r\left(a^{k}\right)=r(a)^{k} .
$$

(d) The radius $r$ vanishes only on nilpotent elements of $\mathcal{A}$.

(e) The radius $r$ is a continuous function on $\mathcal{A}$.

With Theorem 4.4 at hand, we refer to Corollary 2.3 and to the definition of submodulus in order to obtain the following observation which brings us back to the realm of Section 2:

TheOREm 4.5. [G2, Theorem 3.3]. Let $\mathcal{S}$, a subset of a finite-dimensional powerassociative algebra $\mathcal{A}$ over $\mathbb{F}$, be void of nonzero nilpotents and closed under scalar multiplication and under raising to powers. Then:

(a) The radius $r$ is a continuous submodulus on $\mathcal{S}$.

(b) If $\mathcal{S}$ is closed, then $r$ is the only continuous submodulus on $\mathcal{S}$.

By analogy with spectral dominance, we say that a subnorm $f$ on a subset $\mathcal{S}$ of $\mathcal{A}$ is radially dominant if

$$
f(A) \geq r(A) \text { for all } A \in \mathcal{S} .
$$

Hence, combining Theorems 4.5(b) and 2.5, we can state:

TheOREm 4.6. [G2, Theorem 3.4]. Let $\mathcal{S}$, a closed subset of a finite-dimensional power-associative algebra $\mathcal{A}$ over $\mathbb{F}$, be void of nonzero nilpotents and closed under scalar multiplication and under raising to powers. Let $f$ be a continuous subnorm on $\mathcal{S}$. Then $f$ is stable on $\mathcal{S}$ if and only if $f$ is radially dominant on $\mathcal{S}$.

Because of the fundamental role that the radius $r$ plays in Theorem 4.6, it seems useful to determine this radius on various algebras. By formula (4.1), we already know that the radius on $\mathbb{F}^{n \times n}$ is the classical spectral radius. Similarly, appealing to Theorem 4.5(b) and to the first three examples in Section 1, we observe that the radii on $\mathbb{C}, \mathbb{H}$ and $\mathbb{O}$, are given by the modulus functions in (1.2), (1.4) and (1.6), respectively.

We remark that the radii on $\mathbb{C}$ and $\mathbb{H}$ can be also obtained by using the definition in a more direct way. First, revisiting the complex numbers, it has been noticed in [GL3] that the minimal polynomial of $z=\alpha+i \beta \in \mathbb{C}$ is

$$
p_{z}(t)=t^{2}-2 \alpha t+\alpha^{2}+\beta^{2} .
$$

And since the roots of $p_{z}$ are $z$ and $\bar{z}$, we immediately get $r(z)=|z|$.

As for the quaternions, we need an additional result that hardly requires a proof: 
Proposition 4.7. [G2, Proposition 1.1]. Let $\mathcal{A}$ and $\mathcal{B}$ be algebraically isomorphic finite-dimensional power-associative algebras over $\mathbb{F}$, with an algebra isomorphism $\varphi: \mathcal{A} \rightarrow \mathcal{B}$. Let a be an element of $\mathcal{A}$, and let $p_{a}$ and $q_{\varphi(a)}$ denote the minimal polynomials of a in $\mathcal{A}$ and of $\varphi(a)$ in $\mathcal{B}$, respectively. Then $p_{a}=q_{\varphi(a)}$; so the radii of a in $\mathcal{A}$ and of $\varphi(a)$ in $\mathcal{B}$ coincide.

Now, coming back to the quaternions, we recall the well-known mapping

$$
q \rightarrow A_{q} \equiv\left(\begin{array}{cccc}
\alpha & -\beta & -\gamma & -\delta \\
\beta & \alpha & -\delta & \gamma \\
\gamma & \delta & \alpha & -\beta \\
\delta & -\gamma & \beta & \alpha
\end{array}\right), \quad q=\alpha+i \beta+j \gamma+k \delta \in \mathbb{H}
$$

which implies that $\mathbb{H}$ is algebraically isomorphic to the 4-dimensional subalgebra of $\mathbb{R}^{4 \times 4}$

$$
\mathcal{A}=\left\{A_{q}: q \in \mathbb{H}\right\} .
$$

Since for every $q=\alpha+i \beta+j \gamma+k \delta \in \mathbb{H}$ the eigenvalues of the corresponding matrix $A_{q}$ are $\alpha \pm i \sqrt{\beta^{2}+\gamma^{2}+\delta^{2}}$ (each with multiplicity 2), we employ Propositions 4.3 and 4.7 to obtain

$$
r(q)=r\left(A_{q}\right)=\rho\left(A_{q}\right)=\sqrt{\alpha^{2}+\beta^{2}+\gamma^{2}+\delta^{2}}, \quad q=\alpha+i \beta+j \gamma+k \delta \in \mathbb{H} ;
$$

hence $r(q)=|q|$.

Hinging on the concept of radius, one can extend Proposition 2.2 as follows:

THEOREM 4.8. Let $\mathcal{S}$, a closed subset of a finite-dimensional power-associative algebra $\mathcal{A}$ over $\mathbb{F}$, be closed under scalar multiplication and under raising to powers. Let $f$ be a continuous subnorm on $\mathcal{S}$. Then,

$$
\lim _{k \rightarrow \infty} f\left(a^{k}\right)^{1 / k}=r(a) \quad \text { for all } a \in \mathcal{S} .
$$

To prove this result, take a continuous subnorm $g$ on $\mathcal{A}$, and refer to Theorem 2.1 in [G3] by which,

$$
\lim _{k \rightarrow \infty} g\left(a^{k}\right)^{1 / k}=r(a) \quad \text { for all } a \in \mathcal{A} .
$$

Further, let $\nu$ and $\mu$ be positive constants for which (2.1) holds, so that

$$
\mu^{1 / k} g\left(a^{k}\right)^{1 / k} \leq f\left(a^{k}\right)^{1 / k} \leq \nu^{1 / k} g\left(a^{k}\right)^{1 / k}, \quad a \in \mathcal{S} .
$$

Whence, for $a \in \mathcal{S}$,

$$
\lim _{k \rightarrow \infty} f\left(a^{k}\right)^{1 / k}=\lim _{k \rightarrow \infty} g\left(a^{k}\right)^{1 / k}=r(a),
$$


and the theorem is in the bag.

With Theorem 4.8 at our disposal, we conclude the section by recording the following generalization of Proposition 3.3:

Proposition 4.9. Let $\mathcal{S}$, a closed subset of a finite-dimensional powerassociative algebra $\mathcal{A}$ over $\mathbb{F}$, be closed under scalar multiplication and under raising to powers. Let $f$ be a continuous stable subnorm on $\mathcal{S}$. Then $f$ is radially dominant on $\mathcal{S}$.

The proof is almost trivial: Since $f$ is stable, there exists a constant $\sigma>0$ which satisfies (2.3). Thus,

$$
f(a) \geq \lim _{k \rightarrow \infty} \sigma^{-1 / k} f\left(a^{k}\right)^{1 / k}, \quad a \in \mathcal{S},
$$

and (4.2) completes the proof.

We note in passing that the results in this section do not extend to infinitedimensional algebras. For in this case, minimal polynomials usually fail to exist, rendering the notion of radius meaningless.

5. Discontinuous subnorms. We begin our last section by extending Theorem 4.8 and Proposition 4.9 to subnorms which are not necessarily continuous.

Proposition 5.1. Let $\mathcal{S}$, a closed subset of a finite-dimensional powerassociative algebra $\mathcal{A}$ over $\mathbb{F}$, be closed under scalar multiplication and under raising to powers. Let $g$, a subnorm on $\mathcal{S}$, be equivalent to a continuous subnorm on $\mathcal{S}$. Then:

(a) (compare [G3, Theorem 3.1])

$$
\lim _{k \rightarrow \infty} g\left(a^{k}\right)^{1 / k}=r(a) \quad \text { for all } a \in \mathcal{S} .
$$

(b) If $g$ is stable then $g$ is radially dominant.

Again, the proof is brief: By hypothesis, there exist a continuous subnorm $f$ and constants $\mu>0, \nu>0$, such that for all $a$ in $\mathcal{S}$,

$$
\mu^{1 / k} f\left(a^{k}\right)^{1 / k} \leq g\left(a^{k}\right)^{1 / k} \leq \nu^{1 / k} f\left(a^{k}\right)^{1 / k}, \quad k=1,2,3, \ldots ;
$$

thus, taking limits, (a) follows by Theorem 4.8. As for (b), the stability of $g$ ensures a constant $\sigma>0$ for which

$$
g(a) \geq \lim _{k \rightarrow \infty} \sigma^{-1 / k} g\left(a^{k}\right)^{1 / k}, \quad a \in \mathcal{S} ;
$$

and (5.1) concludes the proof. 
To illustrate Proposition 5.1, let $f$ be a continuous subnorm on an arbitrary finite-dimensional power-associative algebra $\mathcal{A}$ over $\mathbb{F}$, and let us fall back on $h_{\kappa}$, the discontinuous subnorm in (1.9). Since $\kappa>1$, we have

$$
f(a) \leq h_{\kappa}(a) \leq \kappa f(a), \quad a \in \mathcal{A}
$$

so by part (a) of the proposition,

$$
\lim _{k \rightarrow \infty} h_{\kappa}\left(a^{k}\right)^{1 / k}=r(a) .
$$

Further, let $f$ be stable. Then, by (2.3) and (5.2),

$$
h_{\kappa}\left(a^{k}\right) \leq \kappa f\left(a^{k}\right) \leq \sigma \kappa f(a)^{k} \leq \sigma \kappa h_{\kappa}(a)^{k}, \quad a \in \mathcal{A}, \quad k=1,2,3, \ldots .
$$

Hence, $h_{\kappa}$ is stable; so by part (b) of the proposition, $h_{\kappa}$ is radially dominant.

To put Proposition 1.5 in perspective, we conclude this paper by noting that there exist discontinuous subnorms on finite-dimensional power-associative algebras which (i) violate formula (5.1), and (ii) are stable without being radially dominant.

For example, let $f$ be a continuous subnorm on $\mathbb{C}$, let $\varphi$ be a discontinuous $\pi$ periodic solution of the functional equation (1.10), and let $g_{\varphi}$ be the ubiquitously discontinuous subnorm in (1.13). Then, Theorem 2.2(a) in [GL2] tells us that

$$
\lim _{k \rightarrow \infty} g_{\varphi}\left(z^{k}\right)^{1 / k}=|z| e^{\varphi(\arg z)} .
$$

Since $r(z)=|z|$ on $\mathbb{C}$, equation (5.3) implies that $g_{\varphi}$ satisfies formula (5.1) precisely for those values of $z$ for which

$$
\varphi(\arg z)=0
$$

And as $\varphi$ is unbounded on any subinterval of $[0,2 \pi)$, the set of points where $g_{\varphi}$ defies formula (5.1) is dense in $\mathbb{C}$, thus settling (i). We observe that since our $\pi$-periodic $\varphi$ must satisfy $\varphi(r \pi)=0$ for every rational number $r,(5.4)$ holds whenever $\arg z$ is a rational multiple of $\pi$. Hence, the set of complex numbers where $g_{\varphi}$ does satisfy formula (5.1) is also dense in $\mathbb{C}$.

Finally, we invoke Theorem 2.2(b) of [GL2], by which $g_{\varphi}$ is stable on $\mathbb{C}$. Yet, as established in [G3], the set of complex numbers where $g_{\varphi}$ fails to be radially dominant is dense in $\mathbb{C}$, so (ii) is settled as well. In fact, it was shown in [G3] that the set where $g_{\varphi}$ majorizes the radius $r$ is also dense in $\mathbb{C}$, implying that no inequality between $g_{\varphi}$ and $r$ is possible. 


\section{REFERENCES}

[AGL] R. Arens, M. Goldberg, and W. A. J. Luxemburg. Multiplicative factors for seminorms II. J. Math. Anal., 170:401-413, 1992.

[Ba] J. C. Baez. The octonions. Bull. Amer. Math. Soc. (N. S.), 39:145-205, 2002.

[Be] C. A. Berger. On the numerical range of powers of an operator. Abstract No. 625-152, Notices Amer. Math. Soc., 12:590, 1965.

[Bo] R. P. Boas, Jr. A Primer of Real Functions. Amer. Math. Soc., Providence, Rhode Island, 1960.

[BSW] F. L. Bauer, J. Stoer, and C. Witzgall. Absolute and monotonic norms. Numer. Math., 3:257-264, 1961

[CS] J. H. Conway and D. A. Smith. On Quaternions and Octonions: Their Geometry, Arithmetic, and Symmetry. A. K. Peters, Wellesley, Massachusetts, 2003.

[D] L. E. Dickson. On quaternions and their generalization and the history of the Eight Square Theorem. Ann. of Math., 20:155-171, 1918-1919.

[FZ] S. Friedland and C. Zenger. All spectral dominant norms are stable. Linear Algebra Appl., 58:97-107, 1984.

[G1] M. Goldberg. Stable norms - from theory to applications and back. Linear Algebra Appl., 404:223-250, 2005.

[G2] M. Goldberg. Minimal polynomials and radii of elements in finite-dimensional powerassociative algebras. Trans. Amer. Math. Soc., 359:4055-4072, 2007.

[G3] M. Goldberg, Radii and subnorms on finite-dimensional power-associative algebras. Linear Multilinear Algebra, 55:405-415, 2007.

[GGL] M. Goldberg, R. Guralnick, and W. A. J. Luxemburg. Stable subnorms II. Linear Multilinear Algebra, 51:209-219, 2003.

[GL1] M. Goldberg and W. A. J. Luxemburg. Stable subnorms. Linear Algebra Appl., 307:89$101,2000$.

[GL2] M. Goldberg and W. A. J. Luxemburg. Discontinuous subnorms. Linear Multilinear Algebra, 49:1-24, 2001.

[GL3] M. Goldberg and W. A. J. Luxemburg. Stable subnorms revisited. Pac. J. Math., 215:1527, 2004.

[GT] M. Goldberg and E. Tadmor. On the numerical radius and its applications. Linear Algebra Appl., 42:263-284, 1982.

[Hal] P. R. Halmos. A Hilbert Space Problem Book. Van Nostrand, New York, 1967.

[Ham] G. Hamel. Eine Basis aller Zahlen und die unstetigen Lösungen der Functionalgleichung $f(x+y)=f(x)+f(y)$. Math. Ann., 60:450-462, 1905.

[HJ] R. Horn and C. R. Johnson. Matrix Analysis. Cambridge University Press, Cambridge, 1985.

[HLP] G. H. Hardy, J. E. Littlewood, and G. Pólya. Inequalities. Cambridge Univ. Press, Cambridge, 1934.

[HR] H. Hahn and A. Rosenthal. Set Functions. Univ. of New Mexico Press, Albuquerque, 1948.

[P] C. Pearcy. An elementary proof of the power inequality for the numerical radius. Michigan Math. J., 13:289-291, 1966.

[Z] A. C. Zaanen. Riesz Spaces II. North Holland, Amsterdam, 1983. 